\title{
Crack growth induced by sonic IR inspection
}

\author{
JOHN C. CHEN ，JACOB KEPHART , KYLE LICK and WILLIAM T. RIDDELL
}

\begin{abstract}
We have developed an experiment to study the propagation of laboratory synthesized fatigue cracks under various controlled conditions during Sonic IR inspection. The experiment provides for good repeatability in testing. The parameters of interest include the initial crack length, load history (stress intensity and load ratio) during crack generation, geometry of the crack, material and also the various conditions involving the ultrasonic excitation source. In general, we find that under typical sonic IR inspection conditions, the initial crack will propagate under sonic IR testing. The crack growth after each inspection event varies and exhibits a distribution in length of propagation. The results show that the average crack propagation decreases with increasing stress intensity factor, and we test two hypotheses about the cause of this. Furthermore, we find that crack propagation is affected by the initial crack length.
\end{abstract}

Keywords: Sonic IR; Fatigue; Crack propagation; Thermal acoustic

\section{Introduction}

Sonic IR is a relatively new, thermal-based, nondestructive evaluation (NDE) method described in its current form by Thomas and colleagues in 2000 (Favro et al. 2000, Thomas et al. 2001). The technique is related to work done previously by other research groups (Mignogna et al. 1981, Rantala et al. 1998). This thermal NDE method is unique compared to other thermal methods since the active energy source is not a heat source, but a sonic one. This method utilizes an ultrasonic gun, typically used for welding plastics, which vibrates at a frequency of 20 or $40 \mathrm{kHz}$ and can deliver acoustical power up to $1 \mathrm{~kW}$. The gun is made up of a piezoelectric stack coupled to a catenoidal horn. The horn is tuned so that the magnitude of vibration is amplified at the horn tip. The horn tip makes intimate contact with the object being inspected. A short burst of high power acoustical energy is injected into an object being studied and various types of defects such as cracks, delaminations and disbonds heat up due to frictional heating. The increased temperature at the defect sites can be detected using a thermal imaging camera. This inspection technique is very fast, taking only a few seconds for the entire inspection process. It is also relatively simple in terms of hardware setup and software requirements. Many uncertainties in the technique exist, however, which inhibit its 
widespread adoption in industry. This paper shows that under certain conditions cracks can grow with this technique and the crack growth is strongly dependent on the prevailing conditions.

\section{Experiment}

\subsection{Sample preparation}

Following ASTM Standard E647, the eccentrically loaded single edge tension (ESET) specimen was selected as our standard. This sample had some advantages over the widely used compact tension (CT) specimen, the main one being that the ESET is much longer, which allowed for easier optical access and inspection. This proved especially useful in this experiment since crack length measurements were performed using a traveling microscope situated very close to the specimen surface. The ESET also had an advantage over the middle tension (MT) specimen in that considerably lower loads were necessary to achieve the equivalent stress intensity factor.

The ESET specimens employed in this study are shown in figure 1, and were fabricated from $3.18 \mathrm{~mm}(0.125 \mathrm{in}$.) thick 2024-T3 aluminum. This material was chosen because it has been widely studied previously (Riddell and Piascik 1998). The sample width was set at $38.1 \mathrm{~mm}$ (1.5 in.), which effectively set all the dimensions of the sample geometry following

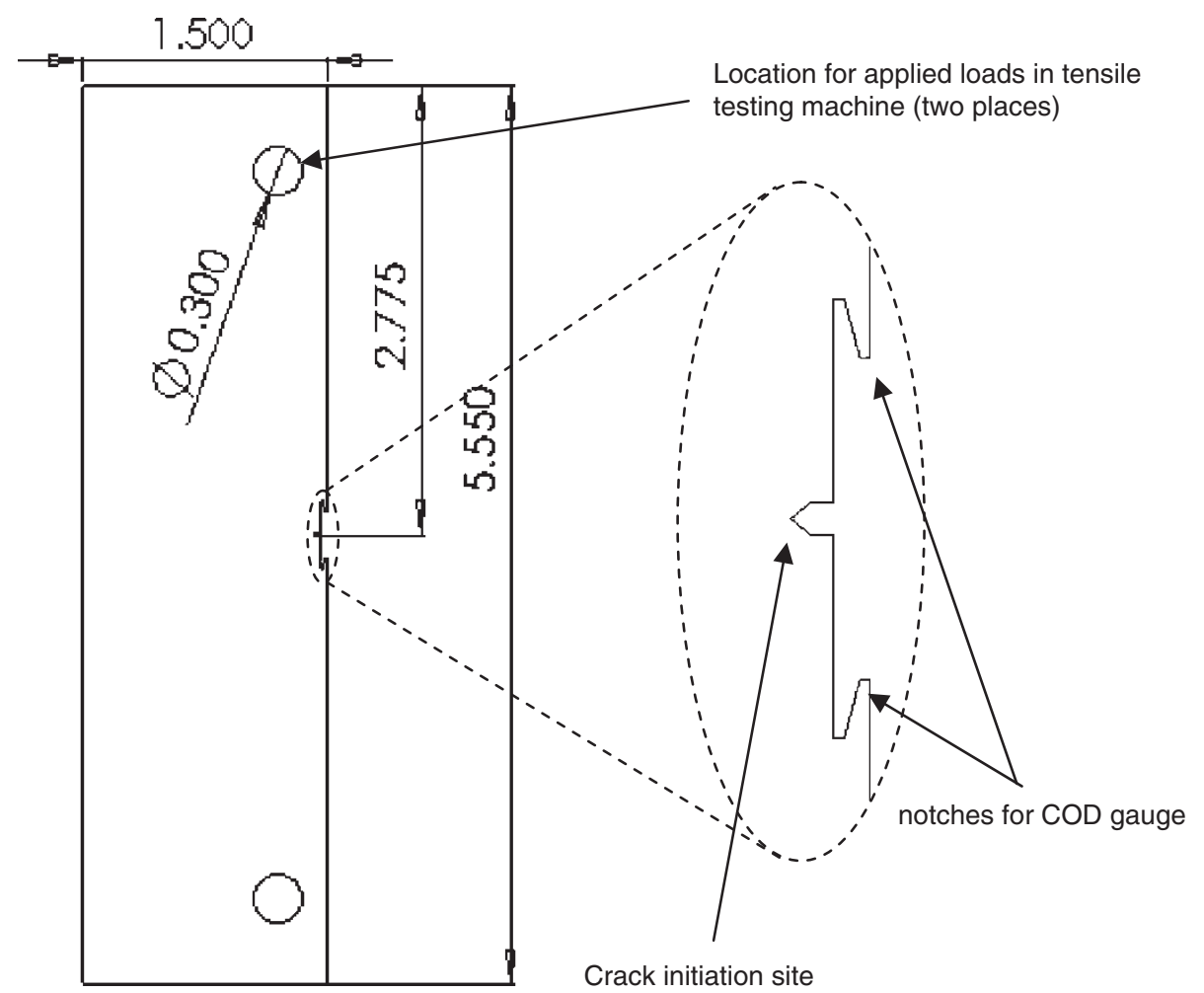

Figure 1. ESET specimen dimensions, in inches and details. The sample thickness is $3.18 \mathrm{~mm}(0.125 \mathrm{in}$.). 
ASTM Standard E647. As the detailed drawing in figure 1 shows, a sharp notch $(0.102 \mathrm{~mm}$ (0.004 in.) radius) was created in one edge of the sample to serve as the crack initiation site. This edge of the sample was also modified by machining two notches above and below the crack initiation site to accept a crack opening displacement (COD) gauge. The COD gauge was used to directly measure the crack opening size (or the sample's compliance) and this measurement was in turn used to control the tensile testing machine by varying the applied load so that a constant stress intensity factor was maintained. A constant stress intensity factor is needed in order to achieve a constant crack growth rate, which in turn is needed to obtain results that are not biased by load history variations. The stress intensity factor at the crack tip can be calculated with the formulae provided in ASTM Standard E647.

The fatigue cracks were created in the ESET specimens using a tensile testing machine by choosing a desired stress intensity factor, $\Delta K$, which is defined as $K_{\max } K_{\min }$, and cyclically loading the sample until the crack length reached the desired value. For the experiments described in this paper (unless otherwise noted), $\Delta K \quad 14 \mathrm{ksi}$-in. ${ }^{0.5}$ with a load ratio, $R\left(K_{\min } / K_{\max }\right)$, of 0.1. Crack lengths between $1.9 \mathrm{~mm}(0.075 \mathrm{in}$.) and $12.7 \mathrm{~mm}(0.500 \mathrm{in})$. were created.

The crack growth rate observed during the creation of a sample with a final crack length of $12.7 \mathrm{~mm}(0.500 \mathrm{in}$.) is shown in figure 2. The initial growth rate is low because for crack ratios, $a / W$ (where $a$ is the crack length and $W$ is the sample width), less than 0.2 , the method used to calculate crack length through the COD gauge over-predicts the length. Therefore, the applied load and stress intensity were lower than required, resulting in the observed low growth rate up to approximately 20,000 cycles, which in this sample corresponded to roughly a crack length of $5 \mathrm{~mm}$. Beyond that, the error is eliminated and a stable crack growth rate is observed. This rate is comparable to that found in previous studies (Levy 1986).

\subsection{Experimental setup}

The sonic IR inspection setup included a Branson ultrasonic welder as the sonic source. The power supply was a Branson $910 \mathrm{M}$ capable of $1 \mathrm{~kW}$ power output and this was coupled to a Branson GK5 handgun converter, which delivered mechanical vibration to the specimen at a frequency of $20 \mathrm{kHz}$. To maintain a constant coupling force, a horizontal sled traveling on two rods was fabricated to hold the handgun (figure 3). This rig clamped the gun in place by its external casing while still allowing its proper operation. A 25 -mm-bore pneumatic

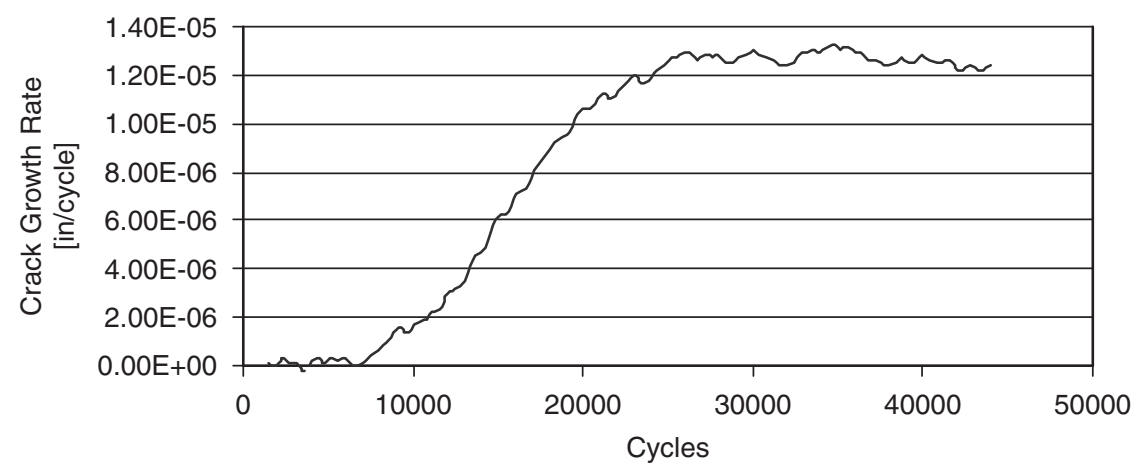

Figure 2. Crack growth rate vs. load cycles in 2024 T3 aluminum. 


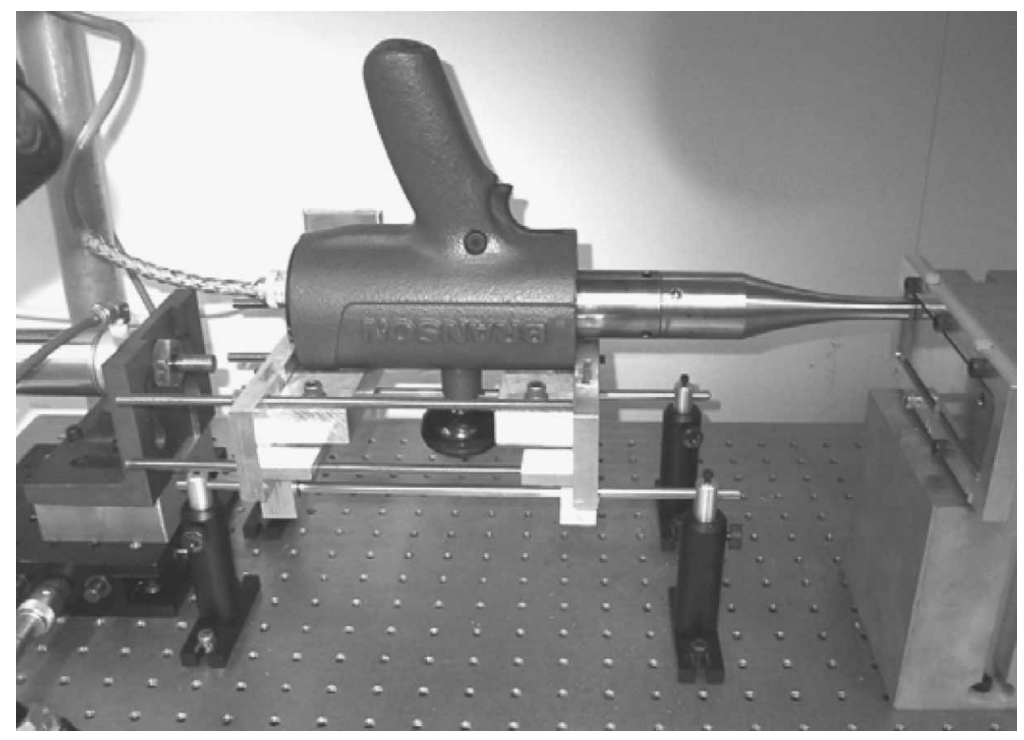

Figure 3. The GK5 handgun secured in sled.

pistoncylinder was attached to the rear of the sled in order to maintain a constant force of the gun against the specimen. We found that small variations in pressure exerted on the handgun can cause significant variations in the experiment, and we took care to ensure the accuracy and constancy of the applied pressure.

The horn tip of the handgun was modified so that it was able to remain securely in place on the specimen without slipping or traveling across the sample surface. The tip was cut back by $1.3 \mathrm{~mm}(0.050 \mathrm{in}$.) so that a protrusion of $7.6 \mathrm{~mm}$ (0.300 in.) in diameter remained, as seen in figure 4. This allowed the protrusion to rest inside either of the loading holes of the ESET specimen. Since fixturing is typically a problem with sonic IR, this design limits motion in all

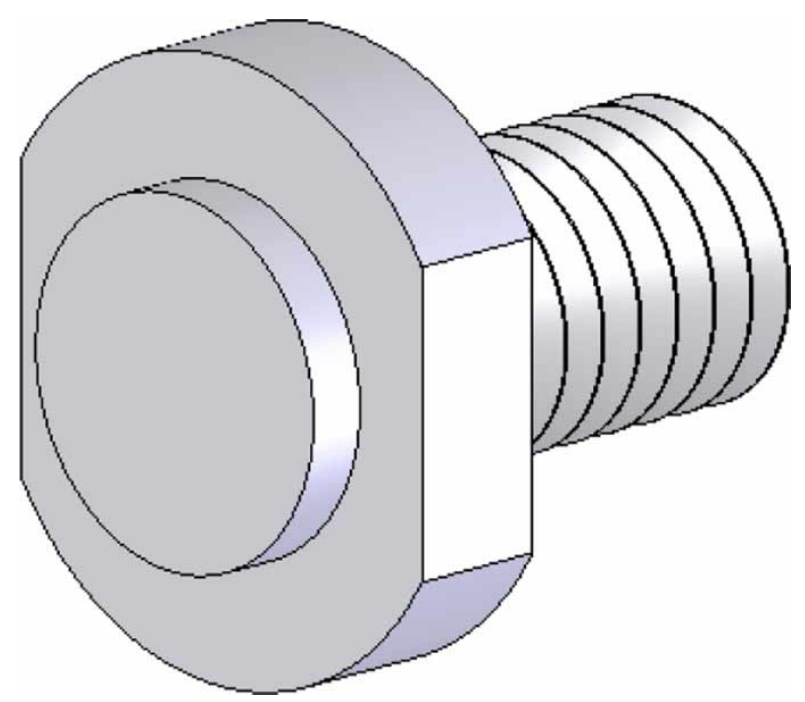

Figure 4. Modified horn tip to disallow slipping across specimen surface. 
directions except in the direction of the axial vibration of the handgun. This limited motion came at the cost of having the ability to only insonate the test sample at one unique location.

The samples being tested were held in a specimen holder so that gross motion in any direction was not possible. The holder (figure 5) had five nylon screws that are adjusted to just make contact with the two long edges of the specimen, thus limiting their vertical motion. The modified horn tip, which sets into one of the loading holes in the ESET specimen, limits specimen travel in the other direction. The sample being tested did not lay in direct contact with the rest of the sample holder; a backing material (high-density polyethylene, $3.2 \mathrm{~mm}(0.125 \mathrm{in}$.) thick) was used between the sample and the sample holder in order to isolate the mechanical vibrations to the sample. Although we did not undertake any measurements to confirm the presence of acoustic chaos (Han et al. 2002,2004) with this experiment, experience and audible signature provided evidence that it was present in these experiments.

Unless otherwise specified, all experiments described in this paper were conducted with the ultrasonic welder set for an insonation period of $250 \mathrm{~ms}$, and the air pressure delivered to the pneumatic piston-cylinder was at $69 \mathrm{kPa}(10 \mathrm{psi})$.

\section{Results and discussion}

Under typical operating conditions for sonic IR inspection, we find that fatigue cracks will propagate, and that the extent of the growth is dependent on the conditions under which the crack was created. Figure 6 shows a photomicrograph of a specimen with an initial crack length of $8.2 \mathrm{~mm}$ ( $0.3209 \mathrm{in}$.) prior to sonic IR inspection, and after it has been subjected to 10 consecutive tests using our standard procedure. In addition to the crack growing to $9.3 \mathrm{~mm}$ $(0.3654 \mathrm{in}$.$) , it can be seen that the surface of the crack is modified by the inspection.$ Specifically, small pieces of the material have been removed along the crack, most likely due to grinding between the two crack surfaces. This hypothesis is supported by evidence of fretting at the crack interface, as will be presented later. We previously demonstrated that cracks of a different geometry, namely those that reside in the center of a thin specimen, and those synthesized in 304 stainless steel and titanium also propagated (Chen et al. 2006).

For our first set of tests, we focused on the effect of stress intensity factor during crack growth. As stated earlier, the stress intensity factor plays a major role in determining the crack growth rate under fatigue loading. Higher stress intensity leads to a faster growth rate, and it also has an effect on the material property at the crack tip. Figure 7 displays a box plot of the data obtained for incremental crack growth after each sonic IR insonation as a function

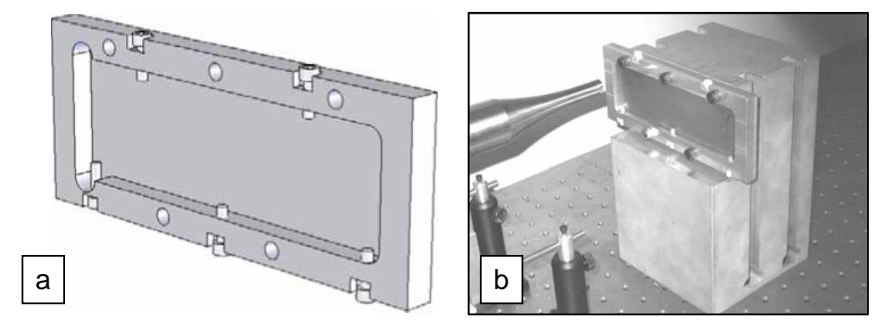

Figure 5. Specimen holder that limits motion of sample during testing. (a) Schematic of the holder. (b) Photograph of the holder and the horn tip of the welder. 


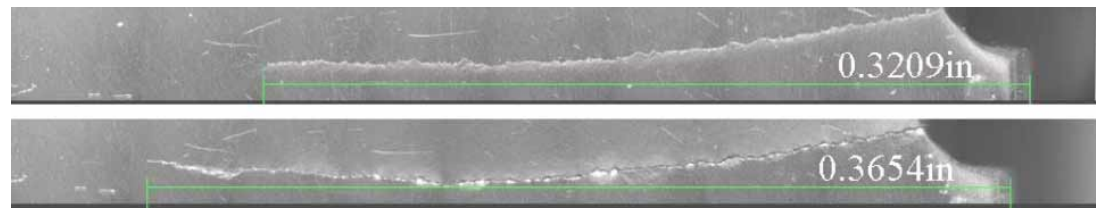

Figure 6. Photomicrographs of a fatigue crack (top) after specimen preparation and (bottom) after 10 consecutive sonic IR tests.

of the stress intensity factor. At each value of $\Delta K$, the box plot is representing 30 total data points. The 30 data points were collected from three ESET specimens created under nominally identical conditions of load ratio, $R$, of 0.1 and initial crack length of $7.6 \mathrm{~mm}$ (0.300 in.). Each specimen was subjected to 10 identical sonic IR tests and the incremental crack growth was measured optically after each test. It may be argued that the 30 data points should not be treated as independent of one another, but our data shows that there is no systematic trend among the data to lead us to believe that the any specific insonation is different from another.

The box plot is used here to depict the distribution of incremental crack growths observed. The solid line inside of the shaded box depicts the median value of the distribution, while the lower and upper edges of the shaded box depict the 25 and 75 th percentiles, respectively. The "whiskers" below and above the shaded box show the 10 and 90th percentiles, respectively, while the lower and upper circles designate the 5 and 95 th percentiles. The dotted line within the shaded box denotes the mean of the distribution.

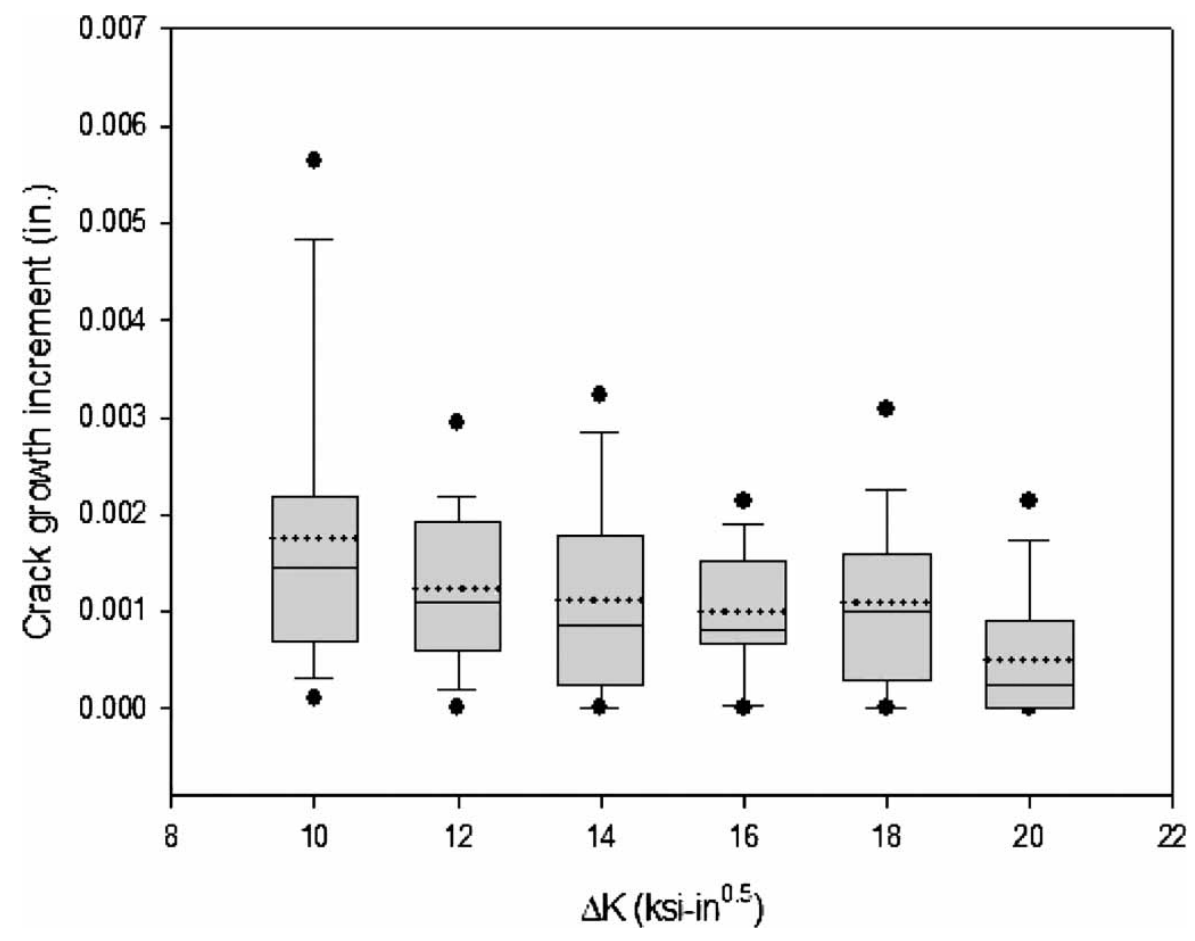

Figure 7. Box plot of observed crack growth increment vs. stress intensity factor, $\Delta K$. 
As can be seen in figure 7, although the data are widely distributed at each $\Delta K$ with most crack growths bunched toward small but non-zero values, the means of the data decreases as the stress intensity factor increases. This observation is further supported by a statistical analysis on the entire data set (again treating all 160 data points as independent of one another) which finds that the data does indeed have a statistically significant $(p<0.05)$ negative slope. We have two hypotheses for this data observation. The first is that higher stress intensity factor creates a larger plastic zone in front of the crack tip, which blunts the crack's growth into the material. The second hypothesis is that the topography of the crack surfaces at various $\Delta K^{\prime}$ 's is affecting the incremental crack growth during sonic IR inspection. While we currently can only indirectly test the first hypothesis (described later), the second could be tested through direct observation and measurement in order to gauge its relative importance.

Figure 8 shows photographs of the ESET specimens for $\Delta K \quad 10$ and $20 \mathrm{ksi}^{-i n^{0.5}}$ after each has been torn apart to expose the crack surfaces. These two conditions were chosen to contrast the difference at the two extremes of stress intensity factors that we examined. Each photograph shows the set of three specimens with the fatigue crack located along the center of each photograph (the left half may be considered the "bottom half" of a sample and the right half the "top half" after it has been rotated $180^{\circ}$ within the plane of the specimen). What is immediately striking about the photographs are the clear signs of fretting that resulted from the sonic IR tests. The fretting patterns show that interfacial grinding within the crack is largely confined to the outer surfaces of the crack, although the specimens at the higher $\Delta K$ do show some fretting on the interior of the cracks.

Even more striking than this observation is the topographical differences in the crack surfaces. Figure 9 shows profile measurements across the crack surfaces for the specimens. Figure 9(a) shows that while the specimens at $\Delta K \quad 10$ display the characteristic cusping at the outer surfaces, the interior profiles of these cracks are very flat. At the higher $\Delta K$ the
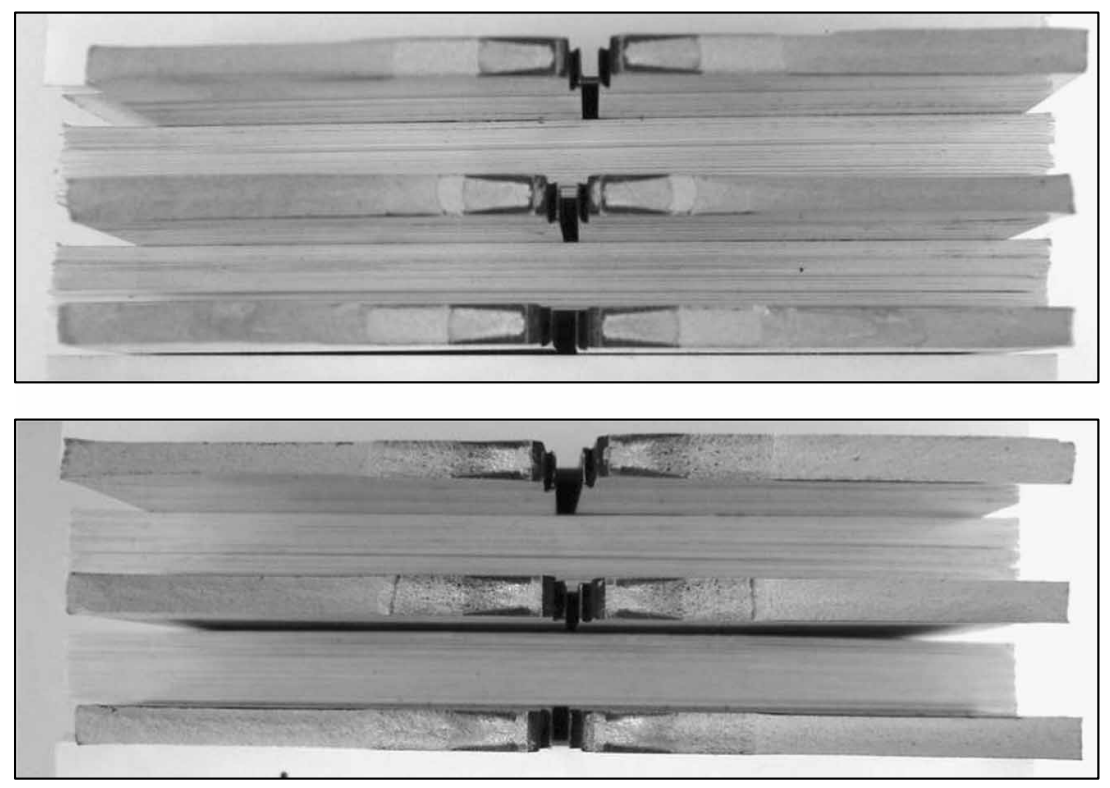

Figure 8. Photographs of the crack surfaces after sonic IR testing. Three samples with cracks grown at (top) $\Delta K=10$, and (bottom) $\Delta K=20 \mathrm{ksi}^{0.5}$. 

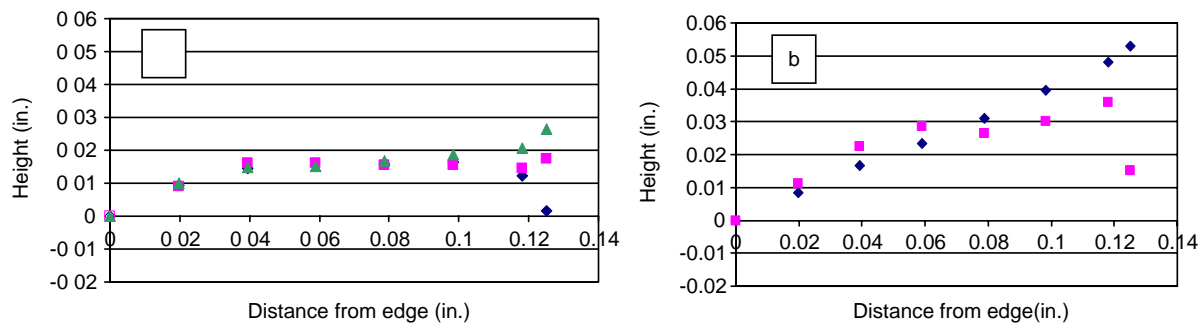

Figure 9. Topographic profile measurements across the crack surfaces for three samples (denoted by different symbols) grown at (a) $\Delta K=10$, and two others grown (b) $\Delta K=20 \mathrm{ksi}^{0.5}$.

profile is dramatically different (figure 9(b)) with either highly curved or slanted surfaces. This type of topography may serve to dissipate more of the ultrasonic energy through frictional heating and thus lead to the observed decrease in incremental crack growth with increasing $\Delta K$.

To further investigate the relative importance of $\Delta K$ and crack topography, we conducted a set of tests to isolate each effect. In these tests, shown in table 1, we compared the average incremental crack growth after 10 tests of each of two specimens (for the purpose of testing for repeatability). (We define "average incremental crack growth" as the average length that the crack extends after undergoing 10 consecutive tests under nominally identical conditions.) The reference-case was of cracks grown at $\Delta K \quad 14, R \quad 0.1$ and initial crack length of $12.7 \mathrm{~mm}$ ( $0.500 \mathrm{in}$.) (table 1, column 2 "regular"). The crack growths observed were relatively small. We next took two nominally identical specimens to the reference pieces and milled away approximately half of the crack after sandwiching the specimens between two sacrificial pieces of aluminum. The average incremental crack growths increased significantly by at least a factor of six (column 3 "milled open") upon testing. This shows that the crack topography and the resulting friction at the crack interface play a strong role in dissipating energy during sonic IR. Next, we again took two reference-case specimens and annealed them at $400^{\circ} \mathrm{C}$ for $2 \mathrm{~h}$. This should have had the effect of eliminating the wake and plastic zone around the fatigue crack. The average crack growth dramatically increased when these specimens were tested (column 4 "heat treated"), again affirming the importance of the plastic zone for inhibiting crack growth. Finally, we took two referencecase specimens and annealed them, followed by 20 load cycles in the tensile testing machine at the maximum load used to create the initial fatigue crack. This processing had the dual effects of eliminating the plastic zone and opening up the crack along its length in order to reduce the frictional rubbing between the crack surfaces (The latter was confirmed by observing the sonic IR testing with the IR camera, which showed that initial heating was isolated at the crack tip.). The crack growth was greatest for this case ("heat treat +20 loadings") as expected since both factors that presumably inhibit crack propagation were eliminated.

Table 1. Average incremental crack growth in each of two samples at the specified conditions.

\begin{tabular}{lllll}
\hline & \multicolumn{1}{c}{ Regular } & \multicolumn{1}{c}{ Milled open } & \multicolumn{1}{c}{ Heat treated } & Heat treat + 20 loadings \\
\hline Specimen 1 & $5.1 \times 10^{3} \mathrm{~mm}$ & $32 \times 10^{3} \mathrm{~mm}$ & $113 \times 10^{3} \mathrm{~mm}$ & $199 \times 10^{3} \mathrm{~mm}$ \\
& $(0.00020 \mathrm{in})$. & $(0.00126 \mathrm{in})$. & $(0.00445 \mathrm{in})$. & $(0.00783 \mathrm{in})$. \\
Specimen 2 & $3.1 \times 10^{3} \mathrm{~mm}$ & $34 \times 10^{3} \mathrm{~mm}$ & $109 \times 10^{3} \mathrm{~mm}$ & $175 \times 10^{3} \mathrm{~mm}$ \\
& $(0.00012 \mathrm{in})$. & $(0.00135 \mathrm{in})$. & $(0.00428 \mathrm{in})$. & $(0.00688 \mathrm{in})$. \\
\hline
\end{tabular}


In our final set of tests, we focused on the effect of the initial crack length on crack propagation. The results obtained are shown in figure 10 for initial crack lengths of 0.075 , $0.100,0.200,0.300,0.400$ and 0.500 in. (1.9, 2.5, 5.1, 7.6, 10.2 and $12.7 \mathrm{~mm}$, respectively), all grown at $\Delta K \quad 14 \mathrm{ksi}^{0 . \mathrm{in}^{0.5}}$ and $R \quad 0.1$. Each box plot again represents the distribution of incremental crack growths measured after each of 10 insonations for three nominally identical specimens (i.e. 30 data points for each initial crack length studied). With the exception of the data at the crack length of $0.400 \mathrm{in} .(10.2 \mathrm{~mm})$, the data showed a decreasing crack growth with increasing initial crack length. The data at the crack length of $0.400 \mathrm{in}$. $(10.2 \mathrm{~mm})$ was reliable (based on additional tests at this condition), so we cannot make a claim about the correlation between crack growth and initial crack length. The cause for the unusually high crack growth at this condition was not clear.

\section{Conclusions}

We have developed a reliable and repeatable sonic IR experiment and used this apparatus to examine the question of whether this NDE technique causes a crack to propagate. Under typical inspection conditions, we found that the cracks do indeed propagate, and that the extent of propagation is dependent on the conditions at which the cracks were created. We emphasize that our findings apply to relative long fatigue cracks synthesized under laboratory conditions.

Cracks created at increasing stress intensity factors tend to propagate less. This finding was shown to be due to two factors. The first is the creation of a larger plastic zone at the crack tip due to the higher stress, which tends to blunt the crack propagation. The second controlling factor is the crack topography, which at high stress intensity factor shows a highly

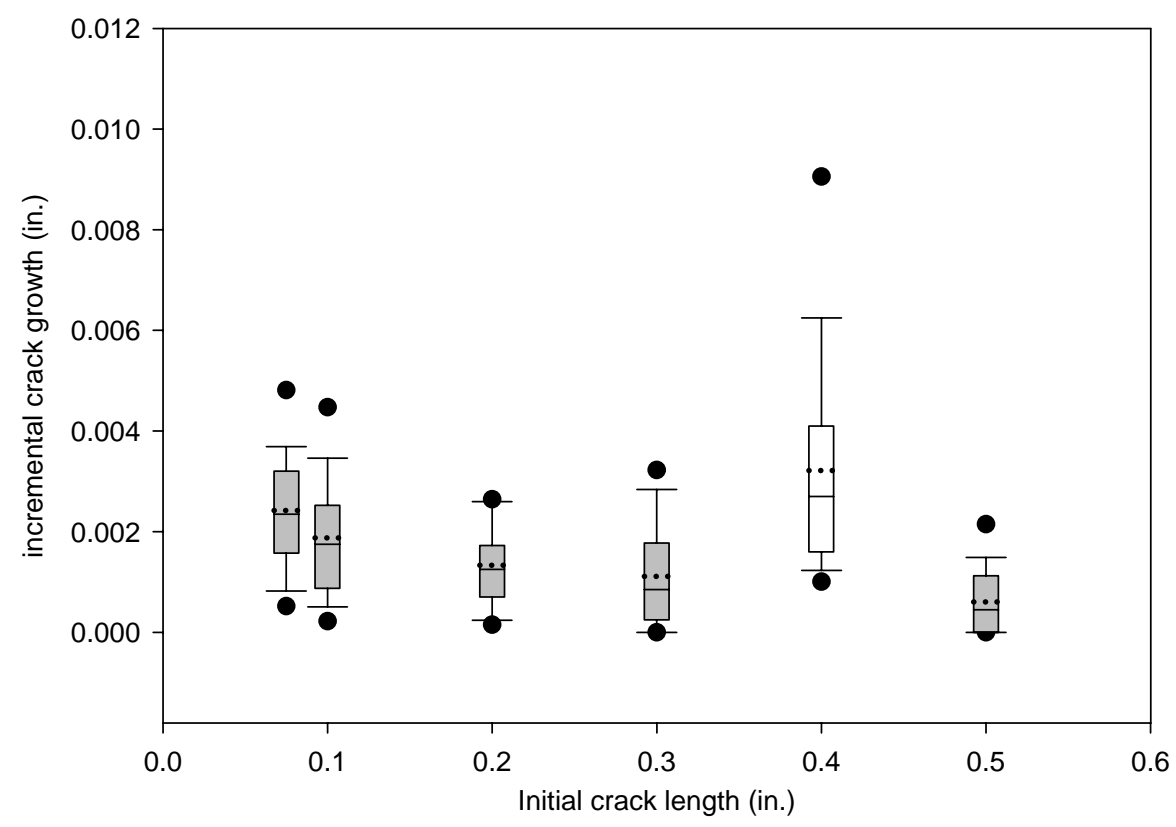

Figure 10. Box plot of observed crack growth increment vs. initial crack length. 
curved or slanted profile. This topographical feature appears to lead to higher energy dissipation at the crack interface, we believe, which reduces the energy available for crack propagation. Finally, our data regarding the incremental crack growth as a function of the initial crack length was inconclusive, though there appears to be some negative correlation between these parameters.

\section{References}

ASTM E647 00, Standard test method for measurement of fatigue crack growth rates, ASTM International 2005. Chen, J.C., Kephart, J. and Riddell, W.T., A parametric study of crack propagation during sonic IR inspection. In Review of Progress in QNDE, 25B, AIP Conference Proceedings edited by D.O. Thompson and D.E. Chimenti, Vol. 820, pp. 1577 1584, 2006 (American Institute of Physics: Melville, NY).

Favro, L.D., Han, X., Ouyang, Z., Sun, G., Sui, H. and Thomas, R.L., Infrared imaging of defects heated by a sonic pulse. Rev. Sci. Instrum., 2000, 71, June 24182421.

Han, X., Li, W., Zeng, Z., Favro, L.D. and Thomas, R.L., Acoustic chaos and sonic infrared imaging. Appl. Phys. Lett., 2002, 81(17), 31883190.

Han, X., Zeng, Z., Li, W., Islam, M.S., Lu, J., Loggins, V., Yitamben, E., Favro, L.D., Newaz, G. and Thomas, R.L., Acoustic chaos for enhanced detectability of cracks by sonic infrared imaging. J. Appl. Phys., 2004, 95(7), 37923797.

Levy, M., Assessment of damage tolerance requirements and analyses tasks II and IV report, AFWAL TR 86 3003, Vol. 4, Raw Test Data 1986.

Mignogna, R.B., Green, R.E., Duke, J.C., Henneke, E.G. and Reifsnider, K.L., Thermographic investigation of high power ultrasonic heating in materials. Ultrasonics, 1981, Vol. 19, 159163.

Rantala, J., Wu, D. and Busse, G., NDT of polymer materials using lock in thermography with water coupled ultrasonic excitation. NDT\&E Int., 1998, 31(1), 4349.

Riddell, W.T. and Piascik, R.S., Stress ratio effects on crack opening loads and crack growth rates in aluminum alloy 2024, NASA Technical Memorandum TM 1998 206929, Langley Research Center 1998.

Thomas, Infrared imaging of ultrasonically excited subsurface defects in materials, US Patent 6,236,049. May 22 et al., 2001. 